\title{
CONCEPTO Y RASGOS DE LA VIOLENCIA DE GÉNERO: PARTICULARIDADES DESDE EL DERECHO DEL TRABAJO ESPAÑOL
}

\author{
JAIME CABEZA PEREIRO* \\ Universidad de Vigo, España
}

\begin{abstract}
RESUMEN: El autor presenta el problema de la violencia de género, contextualizándola en el ámbito de las relaciones laborales. Señala que el derecho del trabajo ha permanecido ajeno a la realidad de la violencia de género; en general, nuestra disciplina ha dado un tratamiento a las cuestiones de género desde la óptica productiva, a saber, igualdad de salarios, mobbing, discriminación, etc., sin prestar atención a las consecuencias y requerimientos que la víctima de violencia de género espera del centro de trabajo. En el artículo, se propone cambiar esta perspectiva unilateral, sirviéndose para ello de las herramientas legislativas que al efecto ya tiene el ordenamiento jurídico español.
\end{abstract}

Palabras clave: Violencia de género, Derecho del Trabajo, discriminacion, igualdad, acoso sexual.

ABSTRACT: The author presents the problem of gender violence, contextualizing it in the field of labor relations. States that labor law has remained oblivious to the reality of gender violence; in general, our discipline has given a treatment of gender issues from the standpoint of production, namely, equal pay, mobbing, discrimination, etc., ignoring the consequences and requirements that the victim of gender violence expects from the workplace. In this paper, we propose to change this unilateral perspective, using for that purpose the legislative tools that the Spanish legal system have.

Key words: Gender violence, Labor law, discrimination, equality, sexual harassment.

\section{INTRODUCCIÓN. LA VIOLENCIA EN EL ÁMBITO PRIVADO}

La violencia de género ha sido una pulsión de las sociedades tradicionales basada en la supremacía del varón, de acuerdo con los patrones socioculturales y socioeconómicos de comportamiento predominantes. La reclusión femenina en el estricto ámbito de las relaciones familiares y del hogar ha producido que todas las formas de agresión masculina hacia su pareja o hacia las mujeres que habitaban bajo su mismo techo se considerarán asuntos absolutamente privados, ubicados en las relaciones familiares e impermeables a la intromisión de las normas jurídicas. En un entorno en el que las formas más abominables de agresión y parricidio con víctimas femeninas han sido banalizadas y socialmente justificadas, cuando no ensalzadas en las más aberrantes frases hechas y cantos populares.

Por supuesto, la sociedad patriarcal en la que hemos habitado -y seguimos habitando, más de lo que comúnmente se acepta- produce un efecto de imposible intercambio entre las distintas funciones vitales y sociales de hombres y mujeres, de tal forma que el espacio doméstico ha constituido la esfera vital de estas, incomunicadas y vulnerables a todos los excesos de aquellos. En tanto que el mundo del trabajo y de las relaciones sociales ha pertenecido en exclusiva al varón. Alrededor de estas presuposiciones se han forjado dos conceptos

\footnotetext{
* Catedrático de Derecho del Trabajo y de la Seguridad Social. Universidade de Vigo, España.
} 
Jaime Cabeza Pereiro / Concepto y rasgos de la violencia de género: particularidades desde el derecho del trabajo español

que han abonado más el campo de los abusos, de las agresiones y de la violencia en general hacia las mujeres: el aislamiento y el estereotipo. Dos conceptos que actúan alternativa o acumuladamente con el mismo efecto de reafimar la supremacía masculina, incluso cuando la mujer se incorpora de forma masiva al mercado de trabajo formal.

Y otro más, que ha sido muy definitorio del Derecho español hasta el cambio de régimen, pero que todavía pervive en algunas manifestaciones. Se trata de la consideración de la mujer como un objeto, más que como un sujeto de derechos. Sus limitaciones a la capacidad de obrar o, en general, el establecimiento de una telaraña de normas jurídicas supuestamente protectoras, pero sobre las que no puede disponer, constituyen ejemplos muy característicos. El derecho y la realidad social han sido las dos palancas que han "cosificado" la identidad femenina, sobre la que se ha dispuesto desde un punto de vista ajeno a la mujer y con connotaciones muy androcéntricas.

Sin perjuicio de que más adelante se reflexione sobre los vínculos entre violencia de género y discriminación, la violencia constituye una forma de evidenciar -quizá la forma más evidente y más característica- la discriminación sistémica de la mujer ${ }^{1}$. Resulta paradójico que el sujeto agente actúa de forma individual y en un contexto muy privado, al menos en la mayoría de las ocasiones, pero que, en realidad, lo haga al amparo de un marco social que propicia sus comportamientos. Por supuesto, este amparo ha disminuido considerablemente en los últimos años, las convicciones no son, en términos generales, las mismas, y el Derecho ha emancipado en parte a la condición femenina. También la Ley Orgánica 1/2004 ha tomado partido decidido contra los agresores. Con todo, las conductas y las pautas de comportamiento son más resistentes al cambio, sin duda porque la violencia está demasiado arraigada como expresión suprema de la dominación del hombre o, en todo caso, como resistencia al cambio.

En este contexto, la violencia producida en el seno familiar constituye la expresión más característica de la violencia de género, porque sin duda es el espacio más privado donde el dominio se ejerce con mayor intensidad. Adopta formas y manifestaciones diferentes, de tal suerte que las más de las veces se coordinan unas y otras. Se conjugan la violencia física con la psíquica, de modo que una y otra destruyen la integrigad física y moral de las mujeres. Pero ambas se tiñen de violencia sexual y sexista, pues la imposición sexual es expresión violenta de dominio y de predominio sobre la mujer, la cual se degrada a objeto de ese dominio. $\mathrm{Y}$, al mismo tiempo, se acompaña de una violencia económica, que sustenta las demás. La subordinación femenina en la familia se agrava por la falta de independencia económica que la hace rehén de su pareja. Y la expone a situaciones de pobreza progresiva a medida que se incrementa la violencia que el hombre ejerce sobre ella. Así sucede, desde luego, en demasiadas ocasiones. La falta de medios la hace también más vulnerable, al cortarle los canales de comunicación con el exterior y la propia posibilidad de separarse de él.

Así se consuman las consecuencias más degradantes de la violencia de género familiar. Tiene una dimensión grande de exclusión social, de enajenación y de aislamiento. La mujer víctima de violencia de género es una persona excluida socialmente, o al menos en riesgo de

Sobre el concepto de discriminación sistémica, SHEPPARD, C. Inclusive equality. The relational dimensions of systemic discrimination in Canada. Montreal, Canadá: McGill-Queen's, University Press, 2010, pp. 21 y ss. 
exclusión. Precisamente por ello es tan importante la dimensión laboral de la trabajadora en estas circunstancias, pues el trabajo se constituye, más que en otras situaciones, en el principal y casi exclusivo contacto social.

\section{LA VERTIENTE LABORAL DE LA VIOLENCIA DE GÉNERO: EL ACOSO. MODELOS JURÍDICOS Y MODELOS SOCIALES}

No se trata ahora de sistematizar las fuentes jurídicas ni tampoco de describir la respuesta del ordenamiento jurídico-laboral sobre el acoso sexual y el acoso por razón de sexo ${ }^{2}$, ambos descritos tanto en la Directiva 2006/54/CE, como en el Derecho interno. Solo, de forma introductoria, conviene recordar los derechos fundamentales y los bienes jurídicamente protegidos más lastimados con estas conductas violentas.

Que el acoso sexual vulnera claramente el principio de igualdad no es algo que quepa atribuir en su reconocimiento a la Directiva 2002/73/CE, ni tampoco a documentos anteriores de la Unión Europea. Debe recordarse que ya el Tribunal Supremo Federal de los Estados Unidos había llegado a semejante conclusión, en 1986, en el muy conocido asunto Vinson $^{3}$. Y que este pronunciamiento, a su vez, se remitía a unas anteriores orientaciones -guidelines- de la Equal Employment Opportunities Commission publicadas en 1980 . Orientaciones que conducen directamente al libro de referencia en esta materia, de C. McKIN$\mathrm{NON}^{4}$, que desarrolló directamente la doctrina de la vertiente discriminatoria del acoso. Puede decirse que el acoso -sobre todo el sexual- constituye una herramienta de dominación del hombre sobre la mujer, la más agresiva sin duda ${ }^{5}$.

En este contexto, la STC 224/1999, de 13 diciembre, afirma asimismo que el acoso sexual vulnera el art. $14 \mathrm{CE}$. Más que afirmarlo, expresa que puede vulnerar el derecho a la no discriminación, porque, "por su frecuencia e intensidad", afecta más a las mujeres que a los hombres. Con todo, no se trata de poner el acento en la trivial argumentación de la sentencia a este respecto, sino en el hecho de que el amparo se otorga por vulneración del art. 10 -derecho a la dignidad-y del art. $18 \mathrm{CE}$-derecho a la intimidad personal-. Probablemente, la lesión de uno y otro mantengan su importancia, a la par que la no discriminación $y$ otro derecho fundamental que ha emergido con fuerza en la doctrina judicial interna, como es el derecho a la integridad moral. Pero la integridad moral constituye el derecho prominente cuando se conoce de conductas de mobbing o, más en general, de violencia en el trabajo. Y es evidente que la violencia sexual y sexista comparten muchos rasgos con las mismas, hasta el punto de poder considerarse una variante, si no una especie de ellas. No obstante, de lo que se trata ahora es de afirmar las particularidades y la autonomía de la violencia ejercida contra la mujer. En este contexto, con toda seguridad, hay que valorizar de nuevo el derecho a la dignidad, pero también, y al mismo nivel, el derecho a la libertad.

\footnotetext{
2 Baste la cita de PÉREZ DEL RIO, T. La violencia de género en el ámbito laboral: el acoso sexual y el acoso sexista. Albacete, España: Bomarzo, 2009.

3 Meritor Savings Bank v. Vinson, 477 US 57, 1986.

4 MCKInnon, C. Sexual Harassment of Working Women. New Haven, Estados Unidos: Yale University Press, 1979.

5 A este tema me he referido más en detalle en "El derecho de la mujer a trabajar en igualdad: apuntes sobre el estado de la cuestión", en: Civitas. Revista Española de Derecho del Trabajo, n 104, 2001, pp. 221 ss.
} 
En efecto, el acoso sexual -y también, al mismo nivel, el sexista- ostentan una connotación muy fuertemente ofensiva. Ambas son conductas degradantes de la personalidad de la víctima, que se identifica individualmente, pero también como miembro de un colectivo secularmente ofendido y degradado. A diferencia de otras conductas de violencia en el trabajo, el acoso en sus vertientes sexual y sexista tiene, desde la perspectiva de la víctima, una importante dimensión colectiva, de la que normalmente carecen otras manifestaciones de violencia que no puedan calificarse de discriminatorias. Se trata de un ataque a la dignidad y a la dimensión femeninas. Lo cual tiene importancia desde el punto de vista de la persona acosada, pero también del acosador, como enseguida va a verse.

Pero antes hay que remarcar cómo también queda afectado el derecho fundamental a la libertad. El acoso sexual -de nuevo también el sexista-constituye una técnica de afirmación del domino masculino sobre el sexo femenino. Lo cual plantea frontalmente uno de los temas más discutidos en la doctrina, que es el del consentimiento de la víctima. Su carácter indeseado se liga, pues, a la voluntariedad de las relaciones personales, que se quiere condicionar a través de conductas que pretenden afirmar la supremacía masculina. Estas conductas, a diferencia de otras, tienen una dimensión más o menos explícita de dominio o de dominación ${ }^{6}$.

Dignidad y libertad son, así pues, dos derechos especialmente lastimados con los acosos sexual y sexista, en particular por el primero de ellos, como conducta más paradigmática de anulación de las personas del sexo femenino como sujetos de derechos. Esta idea ha sido ya suficientemente destacada, y en sí misma resulta poco novedosa. Sin embargo, habría que incidir en una reflexión de carácter más colectivo, que ha quedado algo más en la penumbra.

Me refiero a un análisis tanto de la figura del acosador como de la acosada. En cuanto al primero, podría decirse que hay un cierto empeño de nuestro ordenamiento jurídico en disciplinar al autor como un sujeto individual y desviado de la normalidad. Es decir, en tratarlo como una persona que no encaja en los estereotipos sociales. Cuando tal vez ello no sea del todo así, pues probablemente muchos acontecimientos habría que tratarlos en el marco institucional en que se producen, el cual en no pocas ocasiones propicia el acoso. Es decir, no se corrigen las injusticias estructurales en cuya base se explica el acoso y tampoco se consideran suficientemente. El acoso, como todas las demás manifestaciones de la discriminación por razón de sexo, tiene una innegable dimensión sistémica, en la que el sujeto agente actúa también -aunque no exclusivamente, ni siquiera principalmente en la mayoría de las ocasiones- como miembro de un grupo. El acoso en sus dimensiones sexual y sexista solo se explica en un contexto de relaciones de dominio y de supremacía. Por consiguiente, la solución del problema no se agota en la corrección o en la sanción del sujeto que incurre en estas conductas, ni tampoco en la exigencia de unos elevados estándares preventivos y reparadores a la empresa en cuyo ámbito suceden las mismas. Solo un replanteamiento global de los problemas de dominio y de subordinación que se producen en las estructuras de mando reales -no formales- de las organizadiones productivas puede enfocar globalmente el problema, sin que se afronten tan solo sus consecuencias concretas.

6 Consideraciones al respecto en PÉREZ DEL Rfo, T., op. cit. (n. 2), pp. 32 y ss. 
Desde la perspectiva de las mujeres acosadas, estas reflexiones se observan, si cabe, más nítidamente. En realidad, hay dos contextos generalmente reconocidos como de mayor riesgo. El uno, conformado por situaciones en los que la mujer sufre unas condiciones de aislamiento o de escasa socialización en su trabajo. Encajan en este estereotipo las empleadas domésticas, o las inmigrantes que, por sus condiciones personales, familiares o sociales, o por su origen racial, étnico o religioso, se enfrentan a dificultades de adaptación al medio laboral. $\mathrm{O}$ también las que prestan sus servicios en ambientes fuertemente masculinizados. En términos generales, la progresiva presencia femenina en ámbitos anteriormente reservados a los trabajadores varones disminuye el riesgo de conductas de acoso en la misma proporción en la que va forjando relaciones de solidaridad femenina. Aunque bien es cierto que dicho incremento progresivo viene usualmente acompañado por ráfagas de violencia, casi siempre motivada por el deseo de mantener ciertos statu quo. Pero es claro que la mujer aislada se encuentra en una posición más vulnerable. Cuando más predominen los códigos informales masculinos, más en riesgo se encuentran las mujeres que los padecen.

El otro contexto se caracteriza por un fuerte componente sexual en las relaciones laborales. En él, lo sexista se convierte en sexual y viceversa. No por casualidad se multiplican los episodios de acoso de los que son víctimas mujeres pertenecientes a colectivos tan identificados y consolidados como los de las secretarias, los de las camareras o los de las azafatas de vuelo. En ellos, el trabajo se tiñe de unas connotaciones sexuales al servicio del apetito de los consumidores. Consumidores que pueden ser los clientes, pero que en algún caso -o en bastantes casos- son los propios superiores jerárquicos. En estas situaciones el acoso, más que tolerarse, se nutre de los estándares socialmente admitidos. Precisamente por eso resulta tan importante que ciertos requisitos, realmente inadmisibles, relativos a una uniformidad al servicio de la sexualidad y del sexismo en los lugares de trabajo, vayan, poco a poco, siendo considerados discriminatorios por los órganos judiciales ${ }^{7}$. Bien es cierto que también en estos ámbitos se desarrollan fuertes lazos de solidaridad femenina que constituyen barreras para el acoso, pero insuficientes para resolver todo cuanto hay de sistémico en las estructuras de poder que propician los acosos sexuales y sexistas.

Porque $-\mathrm{y}$ volviendo a la figura del acosador- su existencia encuentra su caldo de cultivo en ciertos prototipos de trabajador que mantienen plena vigencia en la actualidad. Las cualidades que se le presuponen tienen que ver con determinadas características que solo de una forma indirecta pueden tener que ver con la productividad. Puede decirse que se promueve un estereotipo basado en una fuerte virilidad, acompañada de una aparente seguridad en la toma de decisiones y en un componente más o menos inespecífico de supremacía. Es verdad que tales rasgos no son ya universalmente válidos, pero se mantienen con más fuerza de lo que habitualmente se reconoce.

En estos contextos, que la mujer promocione profesionalmente es transgresor, como también lo es que el hombre se manifieste como vulnerable. Tales presuposiciones fomentan todo tipo de situaciones de acoso, distintas de las tradicionalmente jerárquicas en las que la víctima es además subordinada. La dimensión estructural del acoso -sexual y sexista- propi-

7 Es de una importancia fundamental, en el Derecho español, la STS de 19 abril 2011 (recurso de casación n 16/2009). 
Jaime Cabeza Pereiro / Concepto y rasgos de la violencia de género: particularidades desde el derecho del trabajo español

cia un acoso horizontal y también un acoso de los varones sobre sus superiores jerárquicas, en el que, aunque se manifieste como sexual, lo sexista prevalece sobre lo sexual.

\section{LA VIOLENCIA DE GÉNERO EN LA LEY 2004: EL ESCENARIO LABORAL}

\subsection{IDEAS DE ENCUADRE}

La Ley Orgánica de 2004 contempla el ámbito de las relaciones laborales desde una doble perspectiva: como espacio potencialmente violento y como ámbito de socialización de mujeres víctimas de la violencia. Pero, como quiera que entre sus objetivos no se plantea la regulación del acoso -al menos directamente- prevalece la segunda perspectiva, con cierta postergación de la primera.

Es evidente que hunde sus raíces en la Cumbre de Beijing de 1995. Parte, desde luego, de sus grandes principios inspiradores y, en particular, de los tres quizá más basilares: el mainstreaming, el reconocimiento de la violencia de género como una estrategia de dominación y la afirmación de que existe un estrecho parentesco entre violencia y discriminación. No hace falta expresar en estas letras que la Ley de 2004 es la primera técnicamente moderna, desde el punto de vista de que enfoca la violencia de género desde todas las perspectivas, de tal manera que modifica un gran número de leyes, y no parte de una consideración sectorial de ciertos aspectos concretos que había que modificar puntualmente, como hasta entonces habían hecho todas sus predecesoras.

Pero sí que debe destacarse la importancia de focalizar el problema de la violencia, como primer campo de batalla en el que librar la guerra contra la discriminación por razón de sexo. Tiene mucho de simbólico que la LO 1/2004 haya precedido a la LO 3/2007. Es algo más que una casualidad. En el fondo, revela un programa que atiende a la secuencia lógica de la Cumbre de Beijing: la lucha contra la violencia es lo primero. Y no solo porque sea lo más intolerable y lo más indigno, sino porque su erradicación constituye un presupuesto de la igualdad. Abordado el primer asunto, después podrán enfocarse nuevos objetivos. Desde este punto de vista, la LO de 2004 es algo más que una precursora de la de 2007. Constituye, prácticamente, su antecedente necesario. La puesta en cuestión de las relaciones de poder se establece desde su expresión más cruel y simbólica, como es la violencia de género.

Las ideas básicas aparecen, desde luego, en la exposición de motivos de la $\mathrm{LO}$ 1/2004, de 28 diciembre. En ella, al definir a la mujer maltratada, ya se pone el acento en que los patrones sociales y culturales han consolidado unas relaciones de dominio y de enajenación:

"existe ya incluso una definición técnica del síndrome de la mujer maltratada que consiste en "las agresiones sufridas por la mujer como consecuencia de los condicionantes socioculturales que actúan sobre el género masculino y femenino, situándola en una posición de subordinación al hombre y manifestadas en los tres ámbitos básicos de relación de la persona: maltrato en el seno de las relaciones de pareja, agresión sexual en la vida social y acoso en el medio laboral". 
Y se expresa también la necesidad de configurar con contenido amplio el concepto de violencia de género. En el entendimiento de la Ley -art. 1.3-, "comprende todo acto de violencia física y psicológica, incluidas las agresiones a la libertad sexual, las amenazas, las coacciones o la privación arbitraria de libertad".

Destacado, pues, el marco conceptual, los objetivos de la Ley se centran en erradicar la violencia y en configurar el contexto que propicie su erradicación. Y es aquí el punto a partir del cual se deben configurar todas las políticas sectoriales, y también las laborales. Partiendo, pues, de la hipótesis de que el lugar de trabajo es algo más que un espacio potencialmente violento. Es, por encima de otras consideraciones, un ámbito de socialización y de realización, también y sobre todo para las mujeres que sufren violencia en los ámbitos familiares y privados. La relación laboral se tiñe desde esta perspectiva, de una condición emancipadora. Porque la LO 1/2004, es, por encima de todo, una promesa de emancipación de la mujer víctima del predominio violento del hombre, en la que cada sector del ordenamiento jurídico y todos los operadores y operadoras jurídicas tienen que poner su grano de arena.

\subsection{LA ESPIRAL DESPROFESIONALIZADORA DE LA VIOLENCIA}

La violencia sexista que sucede en el ámbito de la pareja condiciona fuertemente la vinculación de las víctimas al mercado de trabajo. Primero, porque deteriora la relación contractual que mantienen, en su caso, con las empresas para las que prestan servicios. No es solo el incremento del absentismo como expresión más visible. Los episodios violentos marcan una ruta de inasistencias, a veces derivadas de bajas, producto de las lesiones físicas o psicológicas que sufren, pero otras veces de los miedos, coacciones y violencias invencibles que deben superar estas mujeres para acudir a sus puestos de trabajo. La violencia sexista tiene mucho de fuerza centrípeta, porque atrae fatalmente a la mujer que la sufre al ámbito de dominación masculina. Ella sufre dificultades para acudir al puesto de trabajo, percibido por el agresor como el espacio en el que no puede desarrollar su posición predominante.

Por supuesto, estas circunstancias empujan a la mujer a la pérdida de su puesto de trabajo. Muchas veces por abandono suyo, a la vista de las condiciones tan poderosas que la rodean. Abandono que se produce formalmente a través de las causas habituales. Pero también por decisiones que le son ajenas, producidas por elementos en apariencia objetivos como una disminución del rendimiento, una bajada en la productividad, una visible falta de implicación en el proyecto de la empresa, o incluso por causas disciplinarias derivadas de inasistencias o impuntualidades u otras que se derivan de su situción de especial sensibilidad. De una manera u otra, con esta ruptura del vínculo laboral el agresor culmina con éxito su «sitio» al espacio de socialización más importante que le quedaba a su víctima. Casi siempre, ante la mirada impasible de la empresa, ajena a estas circunstancias que concibe como extrañas a su ámbito de intereses.

Y es más que la pérdida del puesto de trabajo. Es la pérdida de la conexión con el mercado laboral. No solo por la actitud vigilante del varón y por su tesón en mantenerla en su esfera de control. También, por el propio proceso destructivo de la mujer que sufre estas situaciones. La pérdida de la confianza y de la autoestima, esto es, de la dignidad, genera una fuerte creencia de su incapacidad para todo, y también para volver a trabajar autónomamente de su pareja, en un entorno normal de una empresa. Es, en suma, el proceso hacia la 
Jaime Cabeza Pereiro / Concepto y rasgos de la violencia de género: particularidades desde el derecho del trabajo español

absoluta dependencia y la absoluta servidumbre, en situación de rehén perpetua, sin revisión posible, del acosador, que culmina así su espiral de violencia.

\subsection{El VÍNCULO LABORAL COMO ESPACIO DE COMBATE CONTRA LA VIOLENCIA dE GÉNERO}

Con este planteamiento, el Derecho del Trabajo -y de la Seguridad Social- debe perseguir dos objetivos centrales. El primero, intrínseco a la propia relación laboral, la erradicación de la violencia sexual y sexista de los centros y lugares de trabajo y de las unidades productivas. En estas páginas no se trata de hacer ningún comentario más sobre el acoso discriminatorio, pero sí de poner énfasis en que la empresa debe ser el espacio no violento en el que la víctima de la violencia machista pueda encontrar el contraste con su experiencia personal y el modelo de normalidad.

El otro gran objetivo consiste en revertir la espiral desprofesionalizadora descrita en el apartado anterior, a través de medidas previstas en la normativa estatal, en los convenios colectivos o acuerdos de empresa, o en las iniciativas de responsabilidad social corporativa. Se trata, sobre todo, de mantener la vinculación laboral y la profesionalidad de la víctima. En este esfuerzo, no cabe duda de que la LO 1/2004 ha puesto los primeros peldaños, que deberían ser completados por normas vinculantes producto de la autonomía colectiva o a través de reglas no vinculantes. Pero los distintos sujetos responsables han sido poco diligentes en estas medidas complementarias. Poco se ha hecho además de repetir los mandatos de la Ley, o de afirmar la necesidad de apoyar a las víctimas de esta lacra social. Las normas colectivas -incluidos los planes de igualdad- no han diversificado ni enriquecido especialmente los contenidos de la Ley Orgánica 1/2004, que corre el riesgo de convertirse en el arquetipo de lo posible.

Dicho lo cual, sin ánimo de ahondar ahora en ninguna de las reglas generales o concretas que contiene la Ley Orgánica, bien en su articulado, bien en sus disposiciones adicionales de modificación del Estatuto de los Trabajadores o de otras normas sociales, sí que conviene trazar una especie de "programa" en el que la legislación de protección contra la violencia de género centra su interés.

En primer lugar, hay que considerar un haz de medidas que tienen que ver con el tiempo de trabajo y con su distribución. De una parte, está el tema del absentismo, a la hora de que ciertas inasistencias en que incurra la mujer sistemáticamente agredida puedan merecer la consideración de justificadas, pero no hasta el punto de incentivar unas inasistencias que ahondan más en la violencia. También, el de admitirse horarios más flexibles que le permitan a la mujer una mayor disponibilidad de sus tiempos y una posibilidad más amplia de sortear las dificultades para prestar sus servicios laborales. $O$ también que le posibiliten conciliar su trabajo con otras actividades de recuperación de su integridad física y moral. Por supuesto, hay que contemplar la admisibilidad de incrementos y de reducciones de jornada que puedan servirle para mantener, en la medida de lo posible, su contacto con la empresa o, inversamente, obtener unos mayores recursos que la independicen económicamente del agresor. El objetivo general consiste, pues, en mantener la presencia posible y recomendable de la víctima en la relación de empleo que mantiene con su empresario. 
En segundo lugar, tiene una relevancia indiscutible el apartado de la movilidad, en su doble faceta de funcional o geográfica, así como la modificación de ciertas condiciones de trabajo. Estos cambios deben ponerse al servicio de la mujer sometida a la violencia de género, a los efectos de preservarla alejada del maltratador. En ocasiones puede bastar con un simple cambio de tareas, o con una modificación del lugar de trabajo que no implique cambio de residencia. Probablemente este cambio resulte necesario en no pocas ocasiones. Puede resultar provechoso un cambio de turno. En la adopción de estas medidas, se trata de que la empresa contemple algo ajeno al más inmediato interés económico, técnico, organizativo o productivo, o al menos de que compagine este con las necesidades más perentorias de las mujeres en estas situaciones.

En tercero, se trata de admitir la ausencia cuando no resulte posible la presencia. Pero de cohonestar esta ausencia con el mantenimiento del contrato de trabajo. En este contexto, tienen una importancia grande los permisos, que en estos casos si que pueden definirse con bastante propiedad como derivados de causa de fuerza mayor, y ya sean retribuidos o no retribuidos, en función de lo que establezcan al respecto los convenios colectivos y los planes de igualdad. También ostentan una importancia notable las posibles reducciones de jornada, cuya flexibilidad debería ser amplia como para admitir que la trabajadora pueda mantener un contacto, aunque reducido, con la empresa en la que ha trabajado tradicionalmente. Por supuesto, en ocasiones la necesidad consistirá en la suspensión del contrato de trabajo, revista o no la forma de excedencia. Es verdad que estas medidas, en cuanto más prolongadas en el tiempo, mayor incidencia tendrán en el desarrollo profesional de la trabajadora, pero cuando menos le han de permitir conservar el vínculo laboral y no abandonar definitivamente. Que tales iniciativas puedan tener cierto nivel de protección social, ya sea pública, ya privada, es una tarea que concierne al legislador -como ha tenido en cuenta, aunque solo en relación con la suspensión que da lugar a la prestación por desempleo-, pero también a otros actores sociales.

Y, finamente, está el capítulo extintivo de la relación laboral. Se trata de diferenciar, en la medida de lo posible, la dimisión de la trabajadora víctima de violencia de género de otras dimisiones, formal y materialmente voluntarias por parte del trabajador. En este punto, no es suficiente con que la ley establezca progresivamente medidas en torno al acceso a la prestación por desempleo, o a la prestación por jubilación, sino que se configuren, a través de normas colectivas, determinadas compensaciones económicas en favor de la trabajadora, probablemente a través de fondos de acción social, pero también a través de mecanismos de seguro colectivo o de indemnizaciones a cargo de la empresa. Con las evidentes cautelas de que todo este entramado no se convierta, a la postre, en un mecanismo de incentivo del abandono de la relación laboral y del mercado de trabajo.

\subsection{LOS RETOS DE LA SEGURIDAD SOCIAL Y DE LA ACCION SOCIAL}

En este campo, y por lo que respecta a la Seguridad Social, la labor fundamental que han acometido la Ley Orgánica de 2004 y otras normas sucesivas ha consistido fundamentalmente en adaptar las prestaciones tradicionales a la situación de la mujer víctima de violencia de género. Probablemente, esta sea la técnica más apropiada. En particular, la prestación por desempleo ha sido la más adaptada, mediante la utilización de diversas medi- 
Jaime Cabeza Pereiro / Concepto y rasgos de la violencia de género: particularidades desde el derecho del trabajo español

das. En primer lugar, el establecimiento de una nueva situación legal derivada precisamente de la dimisión del contrato. En segundo, la recuperación de la técnica de la no consunción de las cotizaciones cuando se suceden suspensión y extinción del contrato, con mayor amplitud que en el caso de los expedientes de regulación de empleo y con carácter estructural. Y en tercero, la especial atención del colectivo de las víctimas de la violencia sexista en las acciones de empleabilidad y de búsqueda de nuevo empleo, esta última orientación a caballo entre la protección frente al desempleo y la política de empleo estatal y de las Comunidades Autónomas. Ha sido notable, al respecto, la política de bonificaciones y deducciones de Seguridad Social, así como de subvenciones a la contratación. Y también ha habido cierta labor de ajuste en materia de jubilación, por ejemplo en torno a la anticipada por circunstancias ajenas a la voluntad de la persona interesada, básicamente con ocasión de la Ley $27 / 2011$.

Sin embargo, hay otras prestaciones que no han sido consideradas y que probablemente merecerían tenerse en cuenta desde esta perspectiva, porque pueden tener una incidencia particular en estos colectivos. Por ejemplo, el subsidio por incapacidad temporal, o por maternidad, o también la incapacidad permanente en cualquiera de sus modalidades. $\mathrm{O}$, por supuesto, las lesiones permanentes no invalidantes. En todas ellas, la situación especialmente vulnerable de las víctimas debería de ser considerada a los efectos del otorgamiento de una prestación más proporcionada a su situación de necesidad. Y, asimismo, habría que considerar la protección por muerte y supervivencia. Está bien que se haya suprimido este sinsentido de que el viudo agente de la violencia pudiera percibir una pensión derivada directamente del homicidio, o indirecectamente de las lesiones que infligió a su mujer, con todos los episodios sobrevenidos que aún suceden y se conocen por la opinión pública siete años después de la entrada en vigor de la LO 1/2004. Pero también habría que reorganizar las pensiones de viudedad y orfandad en el caso de un causante que haya incurrido en este tipo de violencia. Porque la viuda y los huérfanos en tales circunstancias están en una situación especialmente cualificada. Sería posible, por ejemplo, equiparar estos casos con los producidos por contingencia profesional, por lo menos a los efectos del acceso y del cálculo de la base reguladora. $\mathrm{O}$ tal vez relajar algo los requisitos para poder accederse a los complementos por mínimos. O cualificar la prorrata de la ex mujer víctima de violencia, aunque no a costa de las demás ex cónyuges.

$\mathrm{Y}$, superponiéndose a esta protección, hay que aludir a todas las medidas de acción social, de las que son responsables principales las Comunidades Autónomas. En torno a esta materia hay medidas muy diversas y ayudas económicas y no económicas. En la práctica, han sido las más decisivas, porque las de Seguridad Social no han servido, básicamente por centrarse en el ámbito de las mujeres titulares de una relación profesional, por cuenta ajena o por cuenta propia. Y sin que se haya dado tampoco el paso de abrir la senda de las prestaciones no contributivas. Lamentablemente, la centralidad de la acción social en esta materia limita considerablemente la seguridad jurídica y económica de las mujeres en estas situaciones. Sobre todo en una época como la actual, de gran ligereza en la consideración de los recortes hacia los más desfavorecidos. Huelga recordar episodios bien conocidos, porque aluden a concretos y piadosos personajes. 


\section{CONCLUSIÓN}

La LO 1/2004 realizó, pues, un muy notable esfuerzo de regulación integral de la situación y de los derechos de las mujeres víctimas de violencia de género. Esfuerzo también considerado por alguna ley posterior, como es el caso de las de reforma de la LO 4/2000, de 11 enero, sobre derchos y libertades de los extranjeros en España. Sin embargo, las normas posteriores solo muy tímidamente ensancharon los derechos a otras relaciones profesionales distintas de las del trabajo por cuenta ajena y dependiente. Es el caso del Estatuto del Trabajador Autónomo, cuyas referencias se limitan a dos reglas concretas relativas a extinciones e interrupciones justificadas de la actividad profesional en el caso de trabajadoras autónomas económicamente dependientes, completadas con las referencias a la situación legal de cese de actividad y acreditación de la misma en la Ley 32/2010. Así pues, debe reconocerse que la regulación laboral en esta materia tiene los límites propios de su carácter laboral (o estatutario-funcionarial), derivados de que no alcanza en su protección a otras relaciones profesionales diferenciadas, asimilables a estos efectos a la del Estatuto de los Trabajadores o del Funcionario Público. En su seno, las mujeres víctimas se mantienen en la trampa de la falta de regulación legal y, por consiguiente, en la situación previa a la LO /2004.

Ello no obstante, la propia existencia de reglas concretas de protección que afectan al desarrollo de la relación laboral es muestra de un Derecho del Trabajo algo más preocupado por la incidencia de circunstancias y acontecimientos que nacen y se desarrollan fuera del propio contrato de trabajo, pero que lo afectan decisivamente. Constituye una manifestación de un Estatuto de los Trabajadores algo más preocupado por situaciones de las personas que trabajan y que son ajenas al mero intercambio de trabajo y salario. Que hasta ahora no se tomaran en consideración constituía, en la práctica, una posición cómplice del ordenamiento jurídico con los agresores.

La LO 1/2004 tiene el doble mérito de ocuparse de estas situaciones, y de hacerlo al margen de cualquier compromiso del Reino de España derivado de su pertenencia a organismos supranacionales. A diferencia de otras materias -v. g., la conciliación de la vida familiar y laboral-, en las que el Derecho interno se mantiene con dificultades en el pelotón de cola de los Estados de la Unión Europea en la ejecución de las normas derivadas, en violencia de género la Ley Orgánica de 2004 legisló en materia laboral sin estar vinculada por ningún tipo de norma originaria o derivada. Lo cual revela, al mismo tiempo, la falta de concernimiento de la Unión Europea, y también la implicación de un Parlamento concreto, animado por el proyecto de un Gobierno concreto, en una fecha y en un lugar determinados.

Y ahora, en una época en la que incluso en el lenguaje político dominante quieren utilizarse eufemismos que no apelen directamente a la violencia de género, hay que defender con militancia esta ley y su desarrollo, porque es una de las piezas legislativas que, verdaderamente, «importan a la gente corriente». Es verdad que corren tiempos difíciles en los que defender el derecho a la no discriminación de la mujer constituye un empeño sometido a la difamación (blacklash) de los mass media y de los círculos de poder. Como casi todos los empeños que merecen la pena. 